\title{
A Formal Outpatient Parenteral Antimicrobial Therapy Program for Patients with Solid Tumors
}

\author{
Alison Robins \\ Baylor College of Medicine
}

\section{Emma Dishner}

Baylor University Medical Center: Baylor University Medical Center at Dallas

\section{Patrick McDaneld}

The University of Texas MD Anderson Cancer Center

\section{Meagan Rowan}

The University of Texas MD Anderson Cancer Center

\section{Jalen Bartek}

The University of Texas MD Anderson Cancer Center

\section{Ying Jiang}

The University of Texas MD Anderson Cancer Center Javier Adachi

The University of Texas MD Anderson Cancer Center Natalie J.M. Dailey Garnes ( $\nabla$ njdailey@mdanderson.org )

The University of Texas MD Anderson Cancer Center https://orcid.org/0000-0002-4016-4929

\section{Research Article}

Keywords: Outpatient parenteral antimicrobial therapy, OPAT, oncology, hospital readmissions, laboratory monitoring, follow-up

Posted Date: May 7th, 2021

DOI: https://doi.org/10.21203/rs.3.rs-420230/v1

License: (c) (i) This work is licensed under a Creative Commons Attribution 4.0 International License. Read Full License 


\section{A Formal Outpatient Parenteral Antimicrobial Therapy Program for Patients with Solid}

Tumors

Alison Robins ${ }^{1,2,4,5}$, Emma Dishner ${ }^{1,2,6}$, Patrick McDaneld ${ }^{3}$, Meagan Rowan ${ }^{1}$, Jalen Bartek ${ }^{1}$, Ying Jiang ${ }^{1}$, Javier Adachi ${ }^{1}$, Natalie J.M. Dailey Garnes ${ }^{1}$

${ }^{1}$ Department of Infectious Diseases, Infection Control \& Employee Health, Division of Internal Medicine, The University of Texas MD Anderson Cancer Center, Houston, Texas, USA

${ }^{2}$ Division of Infectious Diseases, Baylor College of Medicine, Houston, Texas, USA

${ }^{3}$ Division of Pharmacy, The University of Texas MD Anderson Cancer Center, Houston, Texas, USA

${ }^{4}$ Current affiliation: Section of Infectious Diseases, Department of Medicine, Baylor College of Medicine, Houston, Texas, USA

${ }^{5}$ Current affiliation: Infectious Diseases, Medical Care Line, Michael E. Debakey VA Medical Center, Houston, Texas, USA

${ }^{6}$ Current affiliation: Division of Infectious Diseases, Baylor University Medical Center, Dallas, Texas, USA

ORCIDs: Patrick McDaneld 0000-0001-9214-9774; Ying Jiang 0000-0001-6381-1458; Javier Adachi 0000-0001-9721-8804; Natalie J.M. Dailey Garnes 0000-0002-4016-4929

Corresponding Author: Natalie J.M. Dailey Garnes, njdailey@mdanderson.org 


\begin{abstract}
Background and Objectives: Outpatient parenteral antimicrobial therapy (OPAT) for infections has been in use for nearly 40 years, and although it has been found safe and efficacious, its use has been studied primarily among otherwise healthy patients. We aimed to develop and evaluate an OPAT program for patients with cancer, particularly solid tumors.

Methods: We implemented multiple quality improvement interventions between June 2018 and January 2020. We retrospectively and prospectively collected data on demographics, the quality of infectious diseases (ID) physician consultation notes, rates of laboratory test result monitoring, ID clinic follow-up, and 30-day outcomes, including unplanned OPAT-related readmissions, OPAT-related emergency center visits, and deaths.
\end{abstract}

Results: Completeness of ID provider notes improved from a baseline of $77 \%$ to $100 \%$ $(p<.0001)$ for antimicrobial recommendations, $75 \%$ to $97 \%(p<.0001)$ for follow-up recommendations, and $19 \%$ to $98 \%(p<.0001)$ for laboratory test result monitoring recommendations. Completion of laboratory tests increased from a baseline rate of $24 \%$ to $56 \%$ $(p=.027)$. Thirty-day unplanned OPAT-related readmission, ID clinic follow-up, 30-day emergency center visit, and death rates improved without reaching statistical significance. Conclusions: Sustained efforts, multiple interventions, and multidisciplinary engagement can improve laboratory test result monitoring among solid tumor patients discharged with OPAT. Although demonstrating a decrease in unplanned readmissions through institution of a formal OPAT program among patients with solid malignancies may be more difficult compared with the general population, the program may still result in improved safety. 
Keywords: Outpatient parenteral antimicrobial therapy, OPAT, oncology, hospital readmissions, laboratory monitoring, follow-up 


\section{Introduction}

Outpatient parenteral antimicrobial therapy (OPAT), first described in the 1970s, has increasingly been used as an alternative to hospitalization for stable patients with infections not amenable to oral therapy $[1,2]$. OPAT has been associated with improved patient satisfaction, improved quality of life, reduced length of hospital stay, and successful treatment in $88-92 \%$ of cases [3-6]. Despite these benefits, up to 1 in 4 patients are readmitted within 30 days after discharge [7-11]. The Infectious Diseases Society of America initially published guidelines for OPAT in 2004, with updates in December 2018. These guidelines recommend consultation with a physician specializing in infectious diseases (ID), outpatient follow-up, and periodic monitoring of laboratory test results, with the goal of reducing adverse events [12,13]. Since the publication of these guidelines, studies have reported reduced readmissions among patients who follow up with an ID specialist or in an OPAT clinic within 2-4 weeks of hospital discharge $[9,14]$. Lack of laboratory test result monitoring has also been shown to be independently associated with hospital readmission [8].

Although studies and guidelines addressing the safe delivery of OPAT exist, a knowledge gap remains regarding the effectiveness of OPAT in certain patient populations. For example, the effectiveness of OPAT among patients with oncologic diagnoses and receiving antineoplastic therapy requires further exploration. Despite recent developments, cancer therapeutics, or their sequelae, continue to confer varying degrees of immunosuppression, resulting in an increased risk for infection [15-18]. Most studies specifically focused on patients with oncologic diagnoses receiving OPAT have focused on the treatment of febrile neutropenia [19-24]. The current report discusses our quality improvement experience in patients with solid tumors receiving OPAT at a single comprehensive cancer center. 


\section{Methods}

\section{Interventions}

We retrospectively and prospectively reviewed charts of patients with solid tumors at our institution who were discharged with OPAT at the recommendation of an inpatient ID consultation between November 13, 2017, and January 5, 2020. When patients were readmitted while receiving OPAT and the OPAT was continued, we considered this a single OPAT episode. All patients had been admitted to a solid tumor medical or surgical oncology service. We focused on patients with solid tumors as a pilot program with the goal to expand OPAT to additional patient populations. Patients admitted to services focusing on leukemia, lymphoma, myeloma, pediatrics, stem cell transplantation, and oncology hospitalist services were excluded from the current pilot. We also excluded patients discharged to hospice from their otherwise qualifying admission and patients who completed the recommended antimicrobial regimen while admitted or transitioned to oral therapy upon discharge. We included patients discharged to other facilities. At our institution, primary teams write outpatient antimicrobial prescription orders, including for our institutional infusion center, and case management teams arrange care with external outpatient care providers (e.g., a home health agency, home infusion company, or outpatient infusion center).

This quality improvement project was approved by our institutional Quality Improvement Assessment Board and deemed not to be research. We collected pre-intervention data retrospectively by identifying patients who met our inclusion criteria and had an ID consultation between November 13, 2017, and January 8, 2018 (i.e., the pre-intervention phase). During our 
intervention phases, which in total spanned from June 4, 2018, through January 5, 2020, we collected patient information prospectively and retrospectively.

The Plan-Do-Study-Act methodology guided patient safety improvements. By brainstorming and constructing fishbone diagrams, we identified obstacles to patient follow-up in the ID clinic and monitoring laboratory test results. We mapped the OPAT referral process to identify and prioritize opportunities for improvement. We found that many obstacles to patient follow-up could be attributed to unclear ID consultation recommendations. Thus, we initially sought to clarify recommendations by creating a standardized template within our electronic medical record (EMR) software to be used when making these recommendations to ensure their completeness, including antimicrobial name, dose, duration, frequency, follow-up time frame, and monitoring labs (Figure 1). ID providers received weekly reminders regarding the new process during the phase 1 intervention period, which was June 4, 2018, through July 15, 2018. Furthermore, ID providers were asked to securely email our team and outpatient clinical nursing staff the medical record numbers (MRNs) of patients for whom OPAT was recommended to facilitate prospective data collection and transition of care. Concomitantly, outpatient nurses tracked patients for timely follow-up and review of laboratory test results. During the post-phase 1 intervention period, which spanned from July 16, 2018, through January 31, 2019, ID providers received monthly, rather than weekly, reminders to follow these procedures.

Our subsequent intervention phases continued the phase 1 intervention while adding new interventions. Our phase 2 intervention, which spanned from February 1, 2019, through May 14, 2019, focused on engaging the case management teams, so that the process map now also included external home health and infusion agencies and laboratories. For this intervention, we asked the primary inpatient team to copy and paste the standardized template into a case 
management consultation order. For our phase 3 intervention, which spanned May 15, 2019, through January 5, 2020, we asked ID providers to order the recommended recurring laboratory tests to be monitored for the duration of therapy. For both phase 2 and phase 3 interventions, ID providers received monthly reminders to follow these procedures.

In addition to these phased interventions for ID providers, we further involved advanced practice providers (APPs) (i.e., advanced practice nurses and physician assistants practicing ID) in the transition from inpatient to outpatient care. We developed a new APP OPAT clinic, where patients could be seen earlier than their scheduled follow-up appointment with an ID physician. APPs also called patients to query and document side effects and central line complications, as well as to confirm that recommended laboratory tests were being performed. These telephone visits were conducted between hospital discharge and ID clinic follow-up while patients were receiving OPAT.

\section{Data collection}

The inpatient ID consultation team securely emailed MRNs of patients for whom OPAT was recommended to the OPAT team. Data was collected and managed using REDCap (Research Electronic Database Capture) secure, web-based electronic software data capture tools hosted at The University of Texas MD Anderson Cancer Center [25,26]. We reviewed patient data to document admission and discharge dates; date of final ID consultation recommendations; age; sex; type of malignancy; indication for OPAT; patient disposition, including location of OPAT administration; and outcomes. Additionally, we started collecting data on patient-reported adverse outcomes and antimicrobial classes received, as documented in the EMR, during phase 2 
and phase 3 interventions, and after the phase 3 intervention; therefore, this data was available only for a subset of patients.

We monitored the completeness of ID consultation recommendations, focusing on the recommended antimicrobial regimen, follow-up schedule, and laboratory studies. Primary outcomes included completion of laboratory tests and ID clinic follow-up as recommended by the ID physician, measured as percentages of patients completing laboratory tests and follow-up. Secondary outcomes included documented completion of antimicrobial regimens, 30-day unplanned OPAT-related readmissions, OPAT-related emergency center (EC) visits, and deaths.

To measure primary and secondary outcomes, we reviewed patient charts for at least 30 days after discharge and through completion of OPAT if longer than 30 days. Antimicrobial regimen completion, readmissions, EC visits, deaths, and duration of OPAT were determined by documentation in the EMR. Patients who were readmitted during this 30-day period and then discharged to hospice were excluded from the 30-day outcome measure of death, because time of death was not always available in the EMR. Median duration of OPAT was calculated from the time of hospital discharge to completion of OPAT regimen and excluded days when patients were readmitted.

We also evaluated the impact of the program on costs, specifically hospital and provider charges, for 90 days after initial discharge with OPAT.

From January 2019 through June 2020 (i.e., during part of the post-phase 1 intervention, the phase 2 and phase 3 interventions, and after the phase 3 intervention), we administered surveys to a convenience sample of patients discharged with OPAT who returned to the ID clinic for follow-up. The surveys assessed patient opinions regarding communication effectiveness, hospital visits related to side effects, where they received antimicrobials and whether cost was a 
factor in that decision, time spent administering antimicrobials, and location of laboratory test administration.

\section{Statistical analysis}

Categorical variables were compared using the chi-square or Fisher exact test, as appropriate. Continuous variables were compared using the Kruskal-Wallis test (for 5-group comparisons) and the Wilcoxon rank-sum test (for 2-group comparisons). If a significant result $(p<.05)$ was detected for a test that compared 5 groups, pairwise comparisons were performed for each of the 4 intervention phases with the pre-intervention phase, with $\alpha$ levels adjusted using the Holm sequential Bonferroni adjustment to control type I error. All tests were 2-sided with a significance level of 0.05 , except the pairwise comparisons with the $\alpha$ adjustment. Statistical analyses were performed using SAS version 9.3 (SAS Institute Inc, Cary, NC).

\section{Results}

\section{Patient characteristics}

Patient characteristics are listed in Table 1 for each intervention phase. The median age of all patients was 61 years, without any significant difference among intervention phases. The distribution of types of malignancy was mostly similar among phases. The most common underlying malignancies included genitourinary (25.7\%), breast (11.7\%), gynecologic (11.7\%), head and neck (11.2\%), and sarcoma (10.7\%). The most common types of infections treated with OPAT included abscess (32.7\%), bacteremia (28.9\%), skin/soft tissue infections $(25.9 \%)$, intraabdominal infections (18.7\%), and genitourinary infections (15.7\%). OPAT indications were not mutually exclusive, and often intra-abdominal infections and skin/soft tissue infections included 
abscesses. Most patients received OPAT at home either with $(50.4 \%)$ or without $(29.7 \%)$ home health agency assistance, as opposed to in an infusion center (9\%) or long-term acute care facility (4.2\%). Use of home health agency assistance increased over time during the intervention phases, although this trend did not reach statistical significance.

\section{Quality of notes}

Regarding quality of notes, complete antimicrobial recommendations were given for 37 of 48 pre-intervention phase patients $(77 \%)$ compared with all 17 patients $(100 \%)$ during the phase 1 intervention (Table 2). Rates of complete recommendations ranged from $94 \%$ to $100 \%$ during subsequent intervention phases. Regarding follow-up, complete recommendations were given or follow-up was specifically not recommended for 36 of 48 patients $(75 \%)$ during the preintervention phase and for all 17 patients (100\%) during the phase 1 intervention. The rates of complete follow-up recommendations varied from $83 \%$ to $99 \%$ during subsequent intervention phases. The proportion of patients for whom laboratory test recommendations were given, either recommendations for specific laboratory tests or a statement that laboratory tests were not needed, increased from a low of 9 of 48 patients (19\%) during the pre-intervention phase to a maximum of 114 of 116 patients (98\%) during the phase 3 intervention. Changes in all note quality measures were statistically significant $(p<.0001)$.

\section{Primary outcomes}

The pre-intervention phase ID clinic follow-up rate was 55\% (17 of 31 patients), and this increased to $73 \%$ (11/15 patients) during the phase 1 intervention and ranged from $65 \%$ to $76 \%$ during subsequent intervention phases, with no significant change over the project period $(p=$ 
.25 ; Table 2). During the pre-intervention phase, completion of all recommended laboratory tests occurred in 5 of 21 patients (24\%) and reached a maximum of 63 of 111 patients $(57 \%)$

following the post-phase 1 intervention, with a significantly increased completion rate during the post-phase 1 intervention period $(p=.006)$ and phase 3 intervention $(p=.007)$.

\section{Secondary outcomes}

We noted improvement in rates of documented completion of the recommended antimicrobial regimen from $65 \%$ (31 of 48 patients) in the pre-intervention phase to a maximum of $87 \%$ (62 of 71 patients) during the phase 2 intervention (Table 3$)$. We found that 10 of 48 patients (21\%) experienced unplanned OPAT-related readmissions within 30 days of discharge during the preintervention phase, $0 / 17(0 \%)$ during the phase 1 intervention, with a low of $12 / 116(10 \%)$ during the phase 3 intervention for the subsequent phases $(p=.094)$. During the pre-intervention phase, 8 of 48 patients (17\%) experienced OPAT-related EC visits within 30 days of discharge, and OPAT-related EC visits occurred for $0-21 \%$ of patients during the subsequent intervention phases $(p=.26)$. We observed few deaths during the project period, with no statistically significant differences among the intervention phases $(p=.46)$.

Median OPAT duration ranged from 10 to 13 days throughout the phase 1 through phase 3 interventions. Data on adverse events while receiving OPAT and the antimicrobials received were captured for 134 patients (Tables 4 and 5). Forty-six patients (34\%) received 2 or more intravenous antimicrobials, and $71(53 \%)$ received concurrent oral therapy. The most common antimicrobial classes prescribed were daptomycin (37\%), carbapenems (37\%), and cephalosporins (32\%). We also evaluated control charts of costs for the 90 days following the index discharge with OPAT. Although we noted a decrease during the phase 1 intervention 
compared with the pre-intervention phase, this finding was not statistically significant and was not replicated during the phase 3 intervention or after the phase 3 intervention (data not shown).

\section{Patient experience}

Eighty patients provided information on their experiences with OPAT. Patients rated the overall effectiveness of ID provider communication of the plan prior to discharge, on a scale from 1 to 10 (10 being most effective), an average of $8.5(n=76)$. Most patients $(78 / 80,98 \%)$ indicated that they understood why they needed intravenous instead of oral antimicrobials. Only 2 of 80 patients $(3 \%)$ stated that they had to visit the hospital owing to side effects from their intravenous antimicrobials. Eighteen of 80 patients (23\%) reported receiving antimicrobials outside of the home; 6 of these 18 patients (33\%) cited excessive cost as the reason. Overall, 55 of 80 patients $(69 \%)$ reported spending $\leq 2$ hours per day administering or receiving intravenous antimicrobials at home, 18/80 (23\%) spent 2-4 hours per day, 2/80 (3\%) spent >4 hours per day, and 5/80 (6\%) did not respond. Overall, the OPAT process was well received, although some patients reported frustration with some aspects.

\section{Discussion}

To our best knowledge, this is the largest study of OPAT outcomes in patients with solid tumors reported in the literature. We found that the types of infections treated for our patient population differed from previous reports, even from our own institution [2-6, 8-11, 14, 20-22, 27, 28]. Although we noted improvements in the completeness of ID provider recommendations and in laboratory test result monitoring during our project period, improvements in follow-up in the ID clinic and 30-day OPAT-related readmissions did not reach statistical significance. 
Our initial intervention resulted in more complete recommendations by our ID providers regarding follow-up in the ID clinic and laboratory test result monitoring and in improved transitions of care. This intervention, however, did not include efforts to improve our ability to obtain laboratory test results. Because laboratory test result monitoring requires participation from multiple parties external to our ID clinic, our impact was limited without first engaging those stakeholders. Thus, our second quality improvement intervention aimed to achieve broader multidisciplinary engagement to improve acquisition of recommended laboratory test results. Through these combined efforts, we were able to improve laboratory test result monitoring and noted that follow-up in the ID clinic increased, albeit nonsignificantly. Although approximately $50 \%$ of patients still did not have complete laboratory test results available for ID provider review, we were able to reduce the number of patients who had no laboratory test result monitoring throughout the intervention periods. Although multidisciplinary coordination was necessary to improve laboratory test result monitoring, improvement in ID clinic follow-up was facilitated by internal improvements.

Recent studies regarding laboratory test result monitoring have shown that obtaining any, not necessarily weekly, laboratory test results led to a decreased risk for readmission [8]. Thus, our observed reduction in the number of patients with no laboratory test results may help reduce readmissions. That our observed decreased rate of readmissions did not reach statistical significance could reflect the low sample size of this pilot study. However, the degree to which readmissions are preventable among oncology patients has been called into question previously [29]. Other studies have shown that hospital readmissions among oncology patients may be modestly reduced by improving transitions from inpatient to outpatient care with similar interventions to those described here [30]. Those studies showed that even a $4.5 \%$ decrease in 
readmissions could significantly impact costs, suggesting that significant cost savings could result if our observed decrease in readmissions were confirmed by continued analysis.

Nearly one-third of our patients were treated with OPAT for abscesses, a larger percentage than has been previously reported in the literature, where common indications for OPAT include bacteremia and bone and joint infections [2-6, 8-11, 14, 20-22, 27, 28]. Our patient population often had medical comorbidities, which limited their ability to undergo source control procedures and possibly predisposed them to recurrent admissions for ongoing infection. Other notable differences regarding OPAT indications included a higher rate of genitourinary infections requiring OPAT compared with most previous reports. Our patients often had anatomical abnormalities with urinary devices present, likely predisposing them to recurrent and possibly multi-drug-resistant infections, although we did not systematically collect that data.

Regarding costs, although we noted a decrease in charges during the initial phase 1 intervention, it was not sustained during the post-phase 1 intervention or during the phase 3 intervention and after the phase 3 intervention. Given that this project occurred over a >2-year time frame, charges may have increased over time as patients may have received novel, more expensive therapy. A potentially more informative outcome for this population may be time to return to intended oncologic therapy, because therapy may be delayed because of the infection [31]. Increased charges could represent a return to cancer therapy, signaling successful treatment of the infection.

Our study has some limitations. Our findings are unlikely to be generalizable to noncancer patients or even to patients with hematologic malignancies. Additionally, there may be some degree of bias as some solid tumor patients were admitted to the oncology hospitalist service and were excluded from our analysis. Further, during the project period, admission flow 
changed in our institution: patients with certain solid tumor diagnoses, not sarcoma, were admitted to the solid tumor service early in our project period but to our hospitalist service later. This change could have contributed to the increase in the percentage of patients with sarcoma during our project period. However, the impact of the admission flow change on the types of patients treated with OPAT was likely minimal, because the overall indications for OPAT did not change significantly throughout the project period.

Because 30-day outcomes and primary outcomes were evaluated through chart review, we were limited in our ability to capture readmissions and EC visits to outside institutions. Efforts were made to review all notes from patient encounters documenting outside hospital visits along with shared records within the EMR; however, some visits may not have been documented in this way. Thus, we may have underestimated our 30-day rates of EC visits and readmissions. Also, because patients frequently travel to visit our hospital, we were often unable to capture visits with local ID physicians, possibly underestimating rates of ID follow-up. Nonetheless, our population is motivated to follow up within our institution, so these instances are likely few and impact on our conclusions is likely minimal. Over half of the patients were discharged with home health agency assistance, and some patients reported having laboratory tests administered whose results were not noted in the EMR at the time of chart review. These limitations might also result in underestimation of laboratory test result monitoring in our patients, but these laboratory test results are useful only if they are available for review. Furthermore, past studies showing an association between laboratory test result monitoring and decreased readmissions accounted only for patients whose laboratory test results were available for physician review [8]. 
Our patient survey data are limited because they were obtained from a convenience sample of patients who followed up in our ID clinic and because we have no baseline from prior to our OPAT interventions for comparison. Data collection on adverse events and antimicrobial classes was limited in that it was initiated during later intervention phases and could have changed over time. Regarding adverse events, over half of patients received both intravenous and oral therapy, making it difficult to discern whether adverse events were entirely attributable to their intravenous therapy. Chart review did not always reveal whether a central line was in place solely for OPAT or for an alternative indication. When this indication was unclear, central lineassociated bloodstream infections were considered OPAT-related infections, which may have overestimated the number of central line-associated bloodstream infections directly attributed to OPAT.

\section{Conclusion}

Infections among patients with solid tumors discharged with OPAT may differ from those reported among other OPAT patients. Through standardized recommendations, multidisciplinary engagement, and sustained efforts, monitoring of patients with solid tumors discharged with OPAT can be improved. As has been described in the general population, as laboratory test result monitoring improved, we observed somewhat fewer readmissions, suggesting that OPAT programs targeted toward patients with solid tumors are likely to improve the safety of OPAT.

\section{Acknowledgements}


We thank Yadira Lobo for assistance with data entry and Utpala Daftary for assistance with cost control chart analysis. We thank Erica Goodoff, Senior Scientific Editor in the Research Medical Library at The University of Texas MD Anderson Cancer Center, for editing this article.

\section{Declarations}

Funding: not applicable

Conflicts of interest/competing interests: none

Availability of data and material: upon request

Code availability: upon request

Author contributions: Alison Robins: conceptualization, data retrieval and analysis, writing and editing; Emma Dishner: conceptualization, data retrieval and analysis, writing and editing; Patrick McDaneld: conceptualization, data retrieval and analysis, editing; Meagan Rowan: conceptualization, data retrieval; Jalen Bartek: conceptualization, data retrieval and analysis, editing; Ying Jiang: data analysis, writing, editing; Javier Adachi: conceptualization, editing; Natalie Dailey Garnes: conceptualization, data retrieval, data interpretation, writing, editing Ethics approval: approved by local quality improvement board Consent to participate: not applicable Consent for publication: not applicable 


\section{References}

1. Rucker RW, Harrison GM (1974) Outpatient intravenous medications in the management of cystic fibrosis. Pediatrics 54:358-360.

2. Stiver HG, Telford GO, Mossey JM, Cote DD, van Middlesworth EJ, Trosky SK, McKay NL, Mossey WL (1978) Intravenous antibiotic therapy at home. Ann Intern Med 89:690693.

3. Grayson ML, Silvers J, Turnidge J (1995) Home intravenous antibiotic therapy. A safe and effective alternative to inpatient care. Med J Aust 162:249-253.

4. Tice AD (1995) Experience with a physician-directed, clinic-based program for outpatient parenteral antibiotic therapy in the USA. Eur J Clin Microbiol Infect Dis 14: $655-661$.

5. Durojaiye OC, Bell H, Andrews D, Ntziora F, Cartwright K (2018) Clinical efficacy, cost analysis and patient acceptability of outpatient parenteral antibiotic therapy (OPAT): a decade of Sheffield (UK) OPAT service. Int J Antimicrob Agents 51:26-32.

6. Goodfellow AF, Wai, AO, Frighetto L, Marra CA, Ferreira BM, Chase ML, Nicol RE, Leong CA, Tomlinson S, Jewesson PJ (2002) Quality-of-life assessment in an outpatient parenteral antibiotic program. Ann Pharcother 36:1851-1855.

7. Mansour O, Heslin J, Townsend JL (2018) Impact of the implementation of a nursemanaged outpatient parenteral antibiotic therapy (OPAT) system in Baltimore: a case study demonstrating cost savings and reduction in re-admission rates. J Antimicrob Chemother 73:3181-3188. 
8. Huck D, Ginsberg JP, Gordon SM, Nowacki AS, Rehm SJ, Shrestha NK (2014) Association of laboratory test result availability and rehospitalizations in an outpatient parenteral antimicrobial therapy programme. J Antimicrob Chemother 69:228-233.

9. Palms DL, Jacob JT (2020) Close patient follow-up among patients receiving outpatient parenteral antimicrobial therapy. Clin Infect Dis 70:67-74.

10. Means L, Bleasdale S, Sikka M, Gross AE (2016) Predictors of hospital readmission in patients receiving outpatient parenteral antimicrobial therapy. Pharmacotherapy 36:934939.

11. Williams DN, Baker CA, Kind AC, Sannes MR (2015) The history and evolution of outpatient parenteral antibiotic therapy (OPAT). Int J Antimicrob Agents 46:307-312.

12. Tice AD, Rehm SJ, Dalovisio JR, Bradley JS, Martinelli LP, Graham DR, Gainer RB, Kunkel MJ, Yancey RW, Williams DN; IDSA (2004) Practice guidelines for outpatient parenteral antimicrobial therapy. Clin Infect Dis 38:1651-1671.

13. Norris AH, Shrestha NK, Allison GM, Keller SC, Bhavan KP, Zurlo JJ, Hersh AL, Gorski LA, Bosso JA, Rathore MH, Arrieta A, Petrak RM, Shah A, Brown RB, Knight SL, Umscheid CA (2019) 2018 Infectious Diseases Society of America clinical practice guideline for the management of outpatient parenteral antimicrobial therapy. Clin Infect Dis 68:e1-e35.

14. Saini E, Ali M, Du P, Crook T, Zurlo J (2019) Early infectious disease outpatient followup of outpatient parenteral antimicrobial therapy patients reduces 30-day readmission. Clin Infect Dis 69:865-868. 
15. Bupha-Intr O, Haeusler G, Chee L, Thursky K, Slavin M, Teh B (2020) CAR T-cell therapy and infection: a review. Expert Rev Anti Infect Ther. https://doi: $10.1080 / 14787210.2021 .1855143$

16. Gudiol C, Lewis RE, Srati P, Kontoyiannis DP (2021) Chimeric antigen receptor T-cell therapy for the treatment of lymphoid malignancies: is there an excess risk for infection? Lancet Haemotol. https://doi: 10.1016/S2352-3026(20)30376-8

17. Chamilos G, Lionakis MS, Kontoyiannis DP (2018) Call for action: invasive fungal infections associated with ibrutinib and other small molecule kinase inhibitors targeting immune signaling pathways. Clin Infect Dis 66:140-148.

18. Michot JM, Lazarovici J, Tieu A, Champiat S, Voisin AL, Ebbo M, Godeau B, Michel M, Ribrag V, Lambotte O (2019) Haematological immune-related adverse events with immune checkpoint inhibitors, how to manage? Eur J Cancer 122:72-90.

19. Talcott JA, Whalen A, Clark J, Rieker PP, Finberg R (1994) Home antibiotic therapy for low-risk cancer patients with fever and neutropenia: a pilot study of 30 patients based on a validated prediction rule. J Clin Oncol 12:107-114.

20. Johansson E, Bjorkholm M, Wredling R, Kalin M, Engervall P (2001) Outpatient parenteral antibiotic therapy in patients with haematological malignancies. A pilot study of an early discharge strategy. Support Care Cancer 9:619-624.

21. Minotti V, Gentile G, Bucaneve G, Lori AP, Micozzi A, Cavicchi F, Barbabietola G, Landonio G, Menichetti F, Martino P, Del Favero A (1999) Domiciliary treatment of febrile episodes in cancer patients: a prospective randomized trial comparing oral versus parenteral empirical antibiotic treatment. Support Care Cancer 7:134-139. 
22. Rubenstein EB, Rolston K, Benjamin RS, Loewy J, Escalante C, Manzullo E, Hughes P, Moreland B, Fender A, Kennedy K, et al (1993) Outpatient treatment of febrile episodes in low-risk neutropenic patients with cancer. Cancer 71:3640-3646.

23. Paganini H, Gomez S, Ruvinsky S, Zubizarreta P, Latella A, Fraquelli L, Iturres AS, Casimir L, Debbag R (2003) Outpatient, sequential, parenteral-oral antibiotic therapy for lower risk febrile neutropenia in children with malignant disease: a single-center, randomized, controlled trial in Argentina. Cancer 97:1775-1780.

24. Sung L, Feldman BM, Schwamborn G, Paczesny D, Cochrane A, Greenberg ML, Maloney AM, Hendershot EI, Naqvi A, Barrera M, Llewellyn-Thomas HA (2004) Inpatient versus outpatient management of low-risk pediatric febrile neutropenia: measuring parents' and healthcare professionals' preferences. J Clin Oncol 22:39223929.

25. Harris PA, Taylor R, Thielke R, Payne J, Gonzalez N, Conde JG (2009) Research electronic data capture (REDCap) — A metadata-driven methodology and workflow process for providing translational research informatics support. J Biomed Inform 42:377-381.

26. Harris PA, Taylor R, Minor BL, Elliott V, Fernandez M, O’Neal L, McLeod L, Delacqua G, Delacqua F, Kirby J, Duda SN, REDCap Consortium (2019) The REDCap consortium: building an international community of software partners. J Biomed Inform. https://doi: 10.1016/j.jbi.2019.103208

27. Keller SC, Williams D, Gavgani M, Hirsch D, Adamovich J, Hohl D, Gurses AP, Cosgrove SE (2018) Rates of and risk factors for adverse drug events in outpatient parenteral antimicrobial therapy. Clin Infect Dis 66:11-19. 
28. Poretz DM, Eron LJ, Goldenberg RI, Gilbert AF, Rising J, Sparks S, Horn CE (1982) Intravenous antibiotic therapy in an outpatient setting. JAMA 248:336-339.

29. Brown EG, Burgess D, Li C-S, Canter RJ, Bold RJ (2014) Hospital readmissions: necessary evil or preventable target for quality improvement. Ann Surg 260:583-589.

30. Montero AJ, Stevenson J, Guthrie AE, Best C, Goodman LM, Shrotriya S, Azzouqa A-G, Parala A, Lagman R, Bolwell BJ, Kalaycio ME, Khorana AA (2016) Reducing unplanned medical oncology readmissions by improving outpatient care transitions: a process improvement project at the Cleveland Clinic. J Oncol Prac 12:e594-e602.

31. Aloia TA, Zimmitti G, Conrad C, Gottumukalla V, Kopetz S, Vauthey J-N (2014) Return to intended oncologic treatment (RIOT): a novel metric for evaluating the quality of oncosurgical therapy for malignancy. J Surg Oncol 110:107-114. 


\section{Figure Captions}

Fig. 1 Standardized electronic medical record template for infectious diseases provider recommendations, introduced as part of our phase 1 intervention and used in subsequent interventions

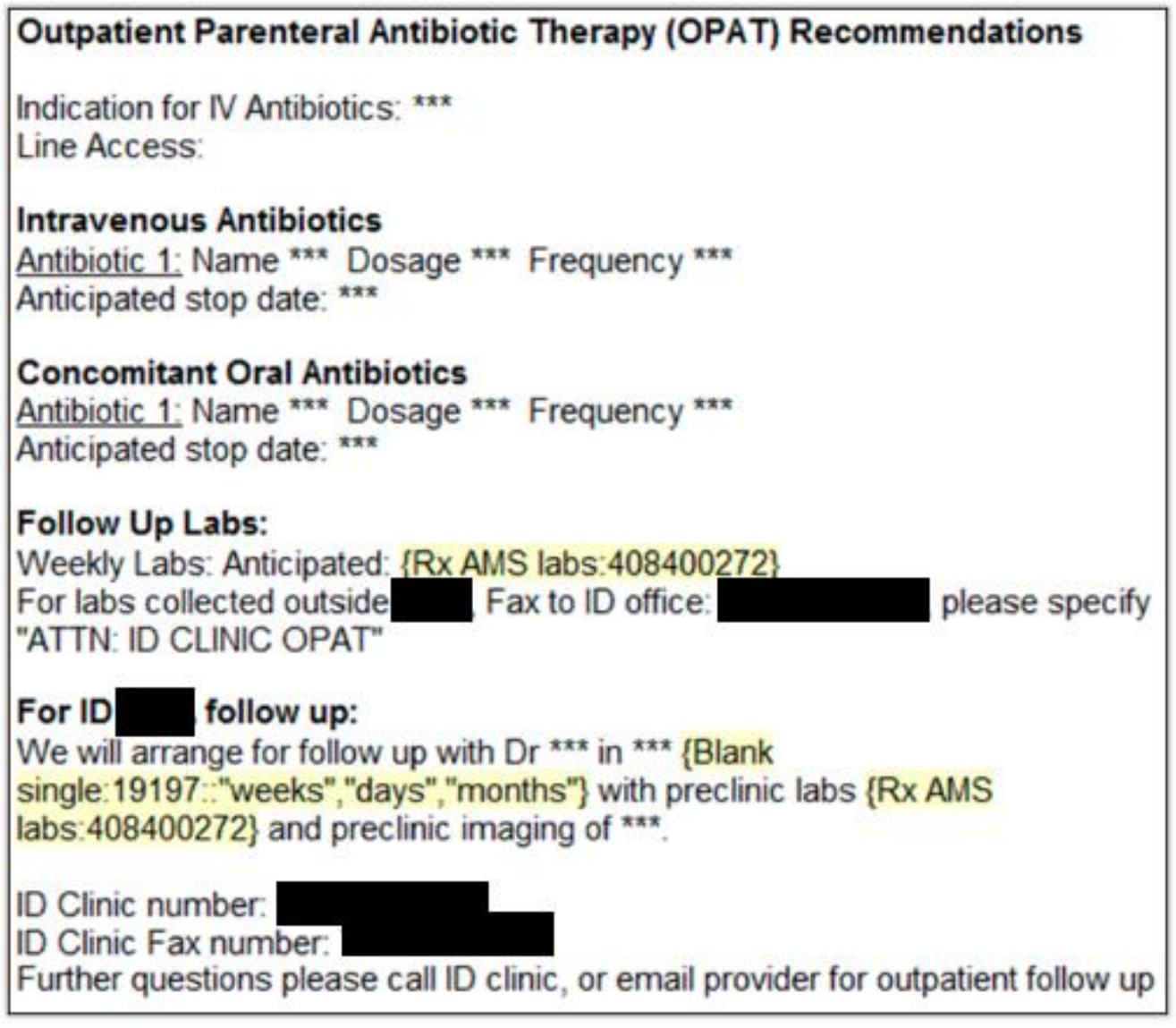


Table 1. Patient demographic information for each intervention phase ${ }^{a}$

\begin{tabular}{|c|c|c|c|c|c|c|}
\hline \multirow[b]{2}{*}{ Characteristic } & \multicolumn{5}{|c|}{ No. $(\%)$} & \multirow[b]{2}{*}{$p$} \\
\hline & $\begin{array}{l}\text { Pre-intervention } \\
\text { phase, } n=48\end{array}$ & Phase 1, n=17 & $\begin{array}{c}\text { Post-phase 1, } \\
n=149\end{array}$ & $\begin{array}{c}\text { Phase 2, } \\
n=71\end{array}$ & $\begin{array}{c}\text { Phase 3, } \\
n=116\end{array}$ & \\
\hline Median age (range) & $\begin{array}{c}63 \text { years }(27-87 \\
\text { years })\end{array}$ & $\begin{array}{c}56 \text { years }(39-76 \\
\text { years })\end{array}$ & $\begin{array}{l}62 \text { years }(21- \\
84 \text { years })\end{array}$ & $\begin{array}{c}57 \text { years }(20- \\
83 \text { years })\end{array}$ & $\begin{array}{c}60 \text { years }(21- \\
90 \text { years })\end{array}$ & .75 \\
\hline Male & $28(58)$ & $6(35)$ & $73(49)$ & $38(54)$ & $63(54)$ & .47 \\
\hline \multicolumn{7}{|l|}{ Type of malignancy } \\
\hline Anal & $0(0)$ & $1(6)$ & $0(0)$ & $0(0)$ & $0(0)$ & .042 \\
\hline Breast & $3(6)$ & $3(18)$ & $21(14)$ & $9(13)$ & $11(9)$ & .49 \\
\hline $\mathrm{CNS}^{\mathrm{b}}$ & $3(6)$ & $2(12)$ & $14(9)$ & $5(7)$ & $9(8)$ & .92 \\
\hline Gastrointestinal & $3(6)$ & $2(12)$ & $26(17)$ & $4(6)$ & $12(10)$ & .064 \\
\hline Genitourinary & $16(33)$ & $7(41)$ & $32(21)$ & $18(25)$ & $30(26)$ & .29 \\
\hline Gynecologic & $9(19)$ & $0(0)$ & $20(13)$ & $8(11)$ & $10(9)$ & .19 \\
\hline Head and neck & $4(8)$ & $0(0)$ & $18(12)$ & $9(13)$ & $14(12)$ & .58 \\
\hline Lung & $3(6)$ & $1(6)$ & $5(3)$ & $1(1)$ & $2(2)$ & .34 \\
\hline Sarcoma & $4(8)$ & $1(6)$ & $8(5)$ & $10(14)$ & $20(17)$ & .025 \\
\hline Skin & $1(2)$ & $0(0)$ & $4(3)$ & $4(6)$ & $4(3)$ & .79 \\
\hline Other & $2(4)$ & $0(0)$ & $5(3)$ & $4(6)$ & $5(4)$ & .91 \\
\hline \multicolumn{7}{|l|}{ OPAT indication } \\
\hline Abscess & $15(31)$ & $4(24)$ & $46(31)$ & $23(32)$ & $43(37)$ & .75 \\
\hline Bacteremia & $15(31)$ & $3(18)$ & $47(32)$ & $17(24)$ & $34(29)$ & .64 \\
\hline CNS infection & $5(10)$ & $4(24)$ & $18(12)$ & $9(13)$ & $10(9)$ & .47 \\
\hline Endovascular infection & $1(2)$ & $3(18)$ & $4(3)$ & $1(1)$ & $2(2)$ & .041 \\
\hline Genitourinary infection & $7(15)$ & $4(24)$ & $22(15)$ & $12(17)$ & $18(16)$ & .91 \\
\hline $\begin{array}{l}\text { Intra-abdominal } \\
\text { infection }\end{array}$ & $7(15)$ & $3(18)$ & $31(21)$ & $12(17)$ & $22(19)$ & .89 \\
\hline Bone/joint infection & $5(10)$ & $1(6)$ & $16(11)$ & $9(13)$ & $11(9)$ & .93 \\
\hline Pneumonia & $4(8)$ & $1(6)$ & $7(5)$ & $1(1)$ & $6(5)$ & .52 \\
\hline Skin/soft tissue infection & $12(25)$ & $2(12)$ & $38(26)$ & $20(28)$ & $32(28)$ & .71 \\
\hline Other & $1(2)$ & $0(0)$ & $4(3)$ & $2(3)$ & $7(6)$ & .64 \\
\hline
\end{tabular}




\begin{tabular}{|c|c|c|c|c|c|c|}
\hline $\begin{array}{l}\text { Home without home } \\
\text { health agency assistance }\end{array}$ & $19(40)$ & $6(35)$ & $51(34)$ & $18(25)$ & $25(22)$ & .081 \\
\hline $\begin{array}{l}\text { Home with home health } \\
\text { agency assistance }\end{array}$ & $21(44)$ & $7(41)$ & $70(47)$ & $34(48)$ & $70(60)$ & .14 \\
\hline Nursing facility & $2(4)$ & $2(12)$ & $11(7)$ & $5(7)$ & $7(6)$ & .81 \\
\hline Long-term acute care & $3(6)$ & $1(6)$ & $2(1)$ & $2(3)$ & $9(8)$ & .06 \\
\hline Outpatient transfusion & $3(6)$ & $1(6)$ & $15(10)$ & $12(17)$ & $5(4)$ & .052 \\
\hline
\end{tabular}

\section{center}

aPre-intervention phase: November 17, 2017-January 8, 2018; phase 1: June 4, 2018-July 15, 2018; post-phase 1: July 16, 2018January 31, 2019; phase 2: February 1, 2019-May 14, 2019; phase 3: May 15, 2019-January 5, 2020.

b Abbreviations: CNS, central nervous system; OPAT, outpatient parenteral antimicrobial therapy. 
Table 2. Primary outcomes during each of the intervention phases ${ }^{\mathrm{a}}$

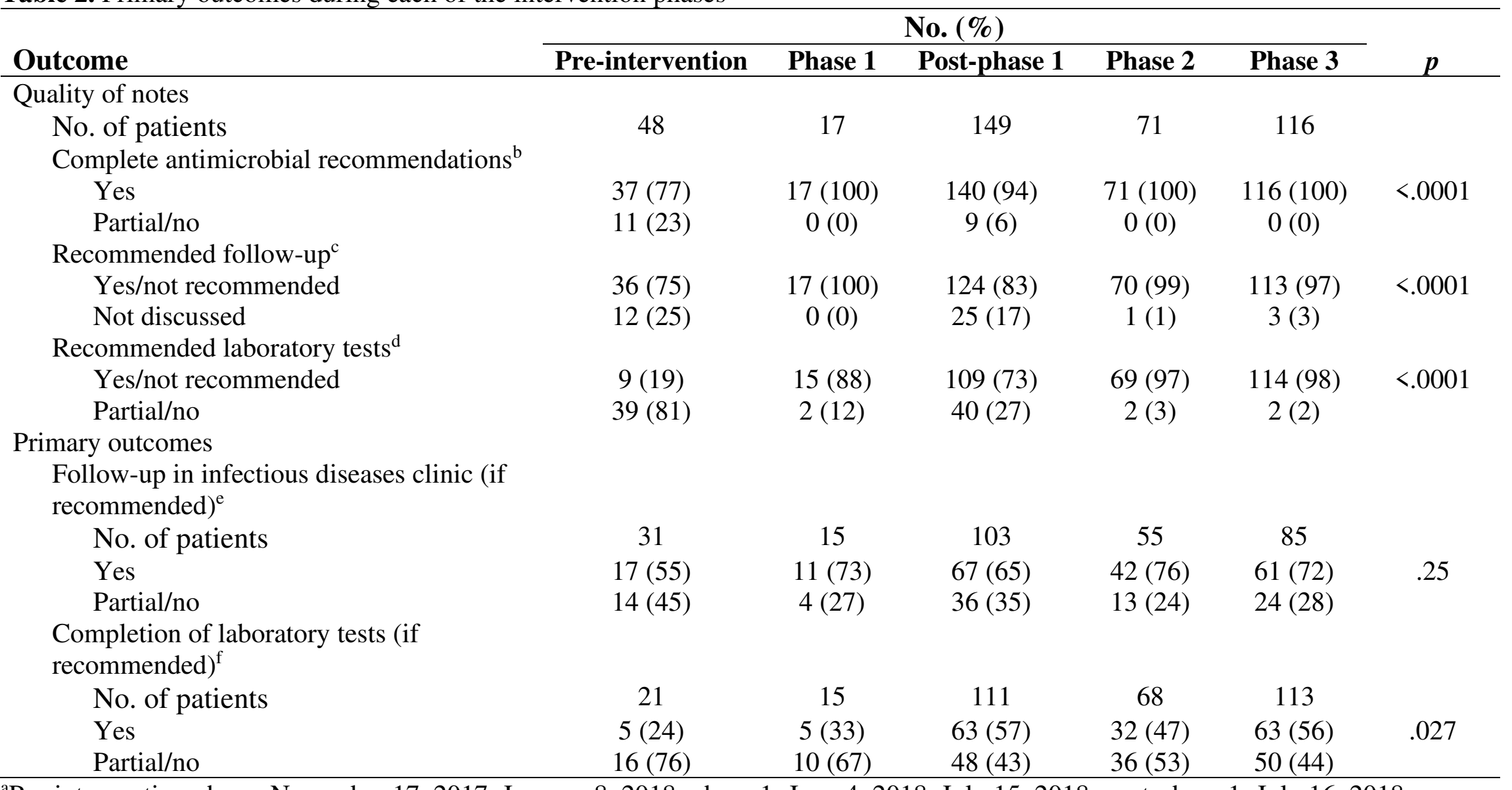

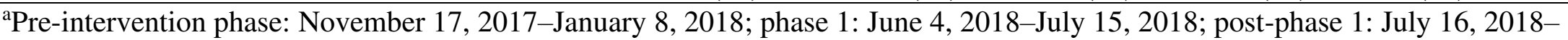
January 31, 2019; phase 2: February 1, 2019-May 14, 2019; phase 3: May 15, 2019-January 5, 2020.

${ }^{\mathrm{b}}$ Yes indicates drug, dose, frequency, and duration were all present; partial indicates 3 of 4 components were present; no indicates 2 or more components were missing.

'Includes provider and timeframe of follow-up.

${ }^{\mathrm{d}}$ Yes indicates laboratory test type, frequency, and contact information for results were all present; partial indicates 2 of 3 components were present; no indicates 2 or more components were missing. 
eYes indicates that patient follow-up was completed within the recommended period; partial indicates that the patient followed up but later than recommended (8-30 days); no indicates that the patient did not follow up within 30 days of the recommended date. The analysis excluded patients for whom the infectious diseases physician either did not provide a recommendation about follow-up or specifically stated that no follow-up was needed. Follow-up by outside infectious diseases providers was considered complete only if we had documentation of this follow-up.

${ }^{\mathrm{f}}$ Yes indicates that the recommended laboratory tests at the recommended frequency were completed and results provided; partial indicates that some of the recommended laboratory tests were completed and results provided; no indicates that no laboratory tests were completed or results provided. The analysis excluded patients for whom the infectious diseases physician either did not provide recommendations about laboratory tests or specifically stated that no laboratory tests were needed. 
Table 3. Secondary outcomes during each of the intervention phases ${ }^{\mathrm{a}}$

\begin{tabular}{|c|c|c|c|c|c|c|}
\hline \multirow[b]{2}{*}{ Outcome } & \multicolumn{5}{|c|}{ No. (\%) } & \multirow[b]{2}{*}{$p$} \\
\hline & $\begin{array}{l}\text { Pre-intervention, } \\
n=48\end{array}$ & $\begin{array}{c}\text { Phase 1, } \\
\mathbf{n}=17\end{array}$ & $\begin{array}{c}\text { Post-phase 1, } \\
n=149\end{array}$ & $\begin{array}{c}\text { Phase 2, } \\
n=71\end{array}$ & $\begin{array}{c}\text { Phase 3, } \\
\text { n=116 }\end{array}$ & \\
\hline Confirmed completion of antimicrobials ${ }^{\mathrm{b}}$ & & & & & & .018 \\
\hline Yes & $31(65)$ & $13(76)$ & $119(80)$ & $62(87)$ & $99(85)$ & \\
\hline Partial/no/unknown & $17(35)$ & $4(24)$ & $30(20)$ & $9(13)$ & $17(15)$ & \\
\hline 30-day outcomes ${ }^{c}$ & & & & & & \\
\hline $\begin{array}{l}\text { Unplanned OPAT-related 30-day } \\
\text { readmission/unknown }\end{array}$ & $10(21)$ & $0(0)$ & $26(17)$ & $14(20)$ & $12(10)$ & .094 \\
\hline Death & $2 / 47(4)$ & $0(0)$ & $5 / 147(3)$ & $0 / 68(0)$ & $2 / 114(2)$ & .46 \\
\hline OPAT-related EC visits/unknown & $8(17)$ & $0(0)$ & $26(17)$ & $15(21)$ & $16(14)$ & .26 \\
\hline
\end{tabular}

apre-intervention phase: November 17, 2017-January 8, 2018; phase 1: June 4, 2018-July 15, 2018; post-phase 1: July 16, 2018January 31, 2019; phase 2: February 1, 2019-May 14, 2019; phase 3: May 15, 2019-January 5, 2020.

${ }^{b}$ Yes indicates that the patient received the correct antimicrobial(s) at the correct dose, frequency, and duration as recommended; partial indicates that the patient received the correct antimicrobial(s) but wrong dose, frequency, or duration; no indicates that the patient received the wrong antimicrobial(s) and/or 2 of the following were wrong: dose, frequency, or duration.

${ }^{c}$ Abbreviations: OPAT, outpatient parenteral antimicrobial therapy; EC, emergency center. Patients enrolled in hospice were excluded from analysis of death as an outcome. For two patients each during the pre-intervention phase and phase 3 intervention, the reason for the EC visit and readmission was unknown. 
Table 4. Adverse events reported during outpatient parenteral antimicrobial therapy, $n=134$

\begin{tabular}{lc} 
Adverse event & No. $(\%)$ \\
\hline $\begin{array}{l}\text { Central line-associated } \\
\text { bloodstream infection }\end{array}$ & $4(3)$ \\
$\begin{array}{l}\text { Line-associated deep venous } \\
\text { thrombosis }\end{array}$ & $3(2)$ \\
Allergic reaction & $3(2)$ \\
Nausea & $15(11)$ \\
Diarrhea & $13(10)$ \\
$\begin{array}{l}\text { Clostridioides difficile infection } \\
\text { Laboratory test result }\end{array}$ & $1(1)$ \\
abnormalities & $10(7)$ \\
Other $^{\mathrm{b}}$ & $13(10)$ \\
\hline
\end{tabular}

a Laboratory test result abnormalities included eosinophilia (2), hyponatremia (1), elevated alkaline phosphatase (1), hypokalemia (1), leukopenia (2; attributed to concomitant oral therapy in one case), and elevated creatine kinase (4).

${ }^{\mathrm{b}}$ Other adverse events included fever/rigors (1), clogged line requiring emergency center visit (2), irritation at peripherally inserted central catheter line site without infection (1), slow flow through peripherally inserted central catheter line with inability to draw blood for laboratory tests (1), myalgias without elevated creatine kinase (1), headache (1), other central nervous system toxic effect (2), urticaria (1; antimicrobial regimen was not changed), neuropathy (1), edema (1), abnormal taste (1), and tinnitus/hearing loss (1). 
Table 5. Intravenous antimicrobials received, $\mathrm{n}=134$

\begin{tabular}{lc}
\hline Antimicrobial class & No. (\%) \\
\hline Carbapenem $^{\mathrm{a}}$ & $49(37)$ \\
Penicillin $^{\mathrm{b}}$ & $9(7)$ \\
Cephalosporin $^{\mathrm{c}}$ & $43(32)$ \\
Vancomycin & $9(7)$ \\
Daptomycin & $49(37)$ \\
New $\beta$-lactam/ $\beta$ - & $5(4)$ \\
lactamase inhibitor & \\
Echinocandin $^{\mathrm{e}}$ & \\
Other $^{\mathrm{f}}$ & $16(12)$ \\
\hline
\end{tabular}

a39 ertapenem, 10 meropenem.

b5 ampicillin, 1 piperacillin/tazobactam, 1 ampicillin/sulbactam, 1 nafcillin, 1 oxacillin.

c 21 ceftriaxone, 11 cefepime, 4 ceftazidime, 7 cefazolin.

d 2 ceftazidime/avibactam, 2 ceftolozane/tazobactam, 1 meropenem/vaborbactam.

${ }^{\mathrm{e}} 15$ caspofungin, 1 anidulafungin.

f 1 aztreonam, 1 doxycycline, 1 eravacycline, 3 tigecycline, 1 polymyxin B, 2 metronidazole, 2 ciprofloxacin, 2 levofloxacin, 1 rifampin, 1 fluconazole. 


\section{Figures}

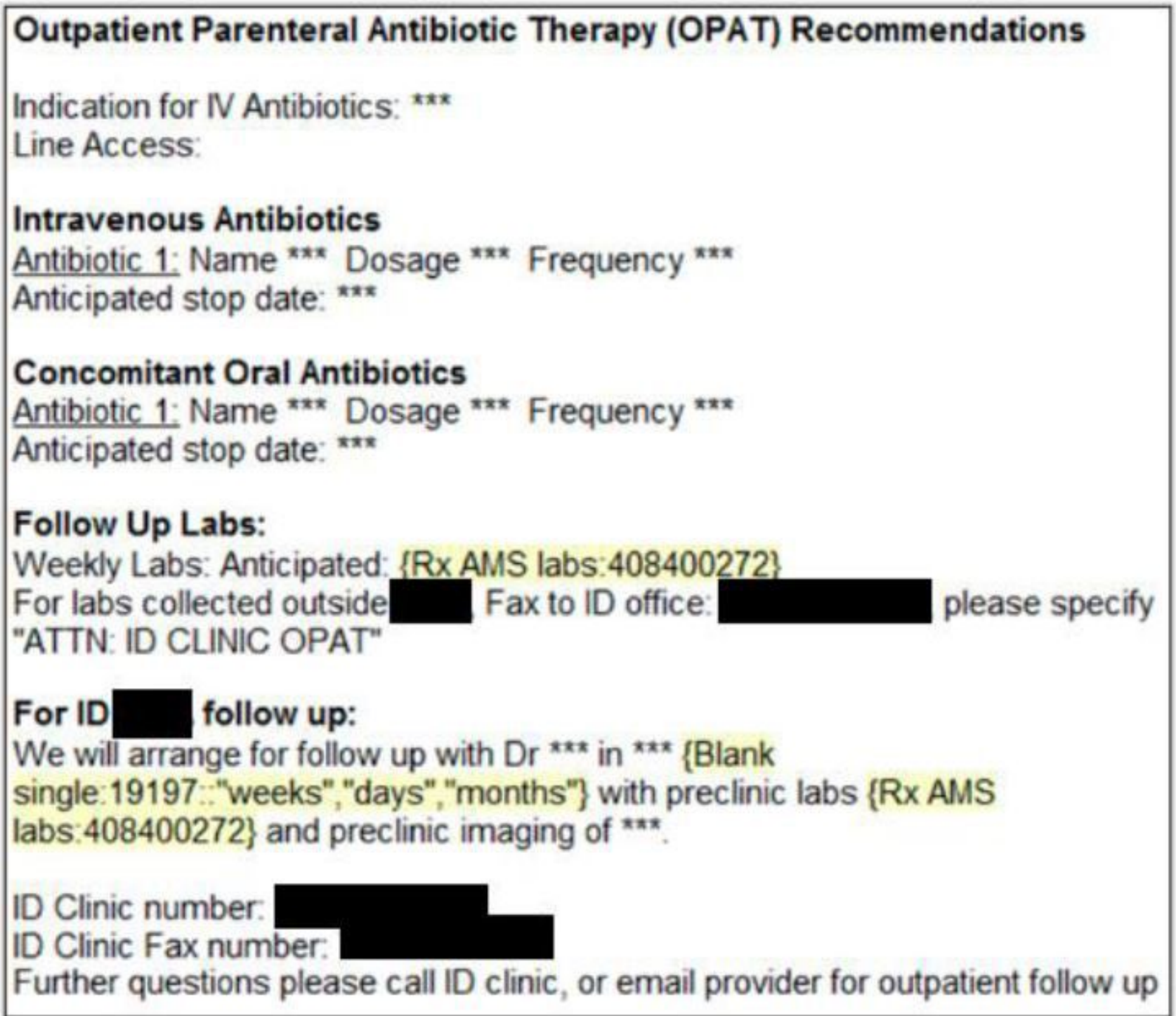

Figure 1

Standardized electronic medical record template for infectious diseases provider recommendations, introduced as part of our phase 1 intervention and used in subsequent interventions 